\title{
Troubleshooting an unusual complication following intrathecal chemotherapy delivered via Ommaya catheter: A case report
}

\author{
DAVID J. MAULER ${ }^{1}$, KENT R. RICHTER ${ }^{1}$, SARAH MERRILL ${ }^{1}$, CRISTINA VALENCIA-SÁNCHEZ ${ }^{2}$, \\ CHANDAN KRISHNA ${ }^{3}$ and MACIEJ M. MRUGALA ${ }^{2}$ \\ ${ }^{1}$ Mayo Clinic Alix School of Medicine, Scottsdale, AZ 85259; ${ }^{2}$ Department of Neurology; \\ ${ }^{3}$ Department of Neurosurgery, Mayo Clinic, Phoenix, AZ 85054, USA
}

Received August 20, 2019; Accepted March 26, 2020

DOI: $10.3892 / \mathrm{mco} .2020 .2032$

\begin{abstract}
The authors report the case of a 39-year-old woman with leukemic meningitis. A right frontal Ommaya reservoir was placed for intrathecal chemotherapy. During and immediately following the first injection of chemotherapy, the patient developed an episode of nausea, emesis, frontal headache and diarrhea. These same symptoms were later elicited during a second and third administration of chemotherapy. Post-placement head computed tomography showed the tip of the catheter projecting approximately $1.5 \mathrm{~cm}$ inferior to the floor of the left frontal ventricle. After a revision of the Ommaya catheter due to suboptimal positioning, subsequent intrathecal chemotherapy administration was tolerated without any of the adverse symptoms previously encountered. The case documents an unusual complication arising from catheter migration in the setting of intrathecal chemotherapy and also demonstrates the value in troubleshooting Ommaya reservoir complications rather than prematurely abandoning its use in favor of lumbar puncture.
\end{abstract}

\section{Introduction}

The Ommaya reservoir has been a treatment option as an alternative to lumbar puncture since the 1960s (1). Previous findings have demonstrated it to be generally well-tolerated in both pediatric and adult settings despite its slightly higher infection risk (2) and other reported complications (3). Patients' comfort and other psychological factors often play a significant role in evaluating its use (4). Ommaya reservoir use for chemotherapy administration has in some settings (e.g., leptomeningeal malignancy) shown higher overall survival compared to intrathecal chemotherapy via lumbar puncture (5). It has been used more extensively as image guidance techniques have advanced (6). Ommaya reservoir is

Correspondence to: Dr Maciej M. Mrugala, Department of Neurology, Mayo Clinic, 5777 E Mayo Blvd, Phoenix, AZ 85054, USA E-mail: mrugala.maciej@mayo.edu

Key words: Ommaya, intrathecal chemotherapy indicated in treatment plans requiring repeated access to the intrathecal space. Applications include treating cancer pain, chronic central nervous system (CNS) infections, leptomeningeal metastasis, and prophylaxis of CNS involvement in acute lymphoblastic leukemia (7).

In the present study, the case of a woman with leukemic meningitis who repeatedly developed severe headache, nausea, emesis, and diarrhea upon administration of intrathecal chemotherapy via an Ommaya reservoir due to catheter migration into brain parenchyma was investigated.

\section{Case report}

The patient was a 39-year-old woman who presented with a three-week history of headaches, dizziness, and night sweats. After noting leukocytosis, the patient was transferred to a tertiary center the following day and a bone marrow biopsy revealed acute lymphoblastic leukemia (8). Her cerebrospinal fluid (CSF) was evaluated and found to have rare blasts. She underwent a lumbar puncture with triple intrathecal chemotherapy (methotrexate + hydrocortisone + cytarabine). Magnetic resonance imaging (MRI) of the brain was subsequently performed and demonstrated mild symmetrical pachymeningeal enhancement which was thought to reflect sequela of the prior lumbar puncture. No abnormal parenchymal or leptomeningeal enhancement was present. The patient developed severe orthostatic headache after the lumbar puncture, and the decision was made to place an Ommaya reservoir with concern that repeated lumbar punctures would increase her risk of CSF leak with persistent headaches secondary to intracranial hypotension.

Three days later, she began oral dasatinib and dexamethasone therapy per treatment protocol. The following day, a right frontal Ommaya reservoir was placed with the aid of electromagnetic intraoperative stealth guidance (6). The intraventricular catheter was inserted into the right frontal horn and clear CSF could be seen coming out. The opening pressure was approximately 5-6 $\mathrm{mmHg}$. The Ommaya reservoir was then tested with a small blunt needle and was found to be working optimally. No intraoperative complications were reported. Follow-up computerized tomography (CT) head showed the new right-sided Ommaya reservoir with the tip of the catheter projecting approximately $1.5 \mathrm{~cm}$ inferior to the floor of the 


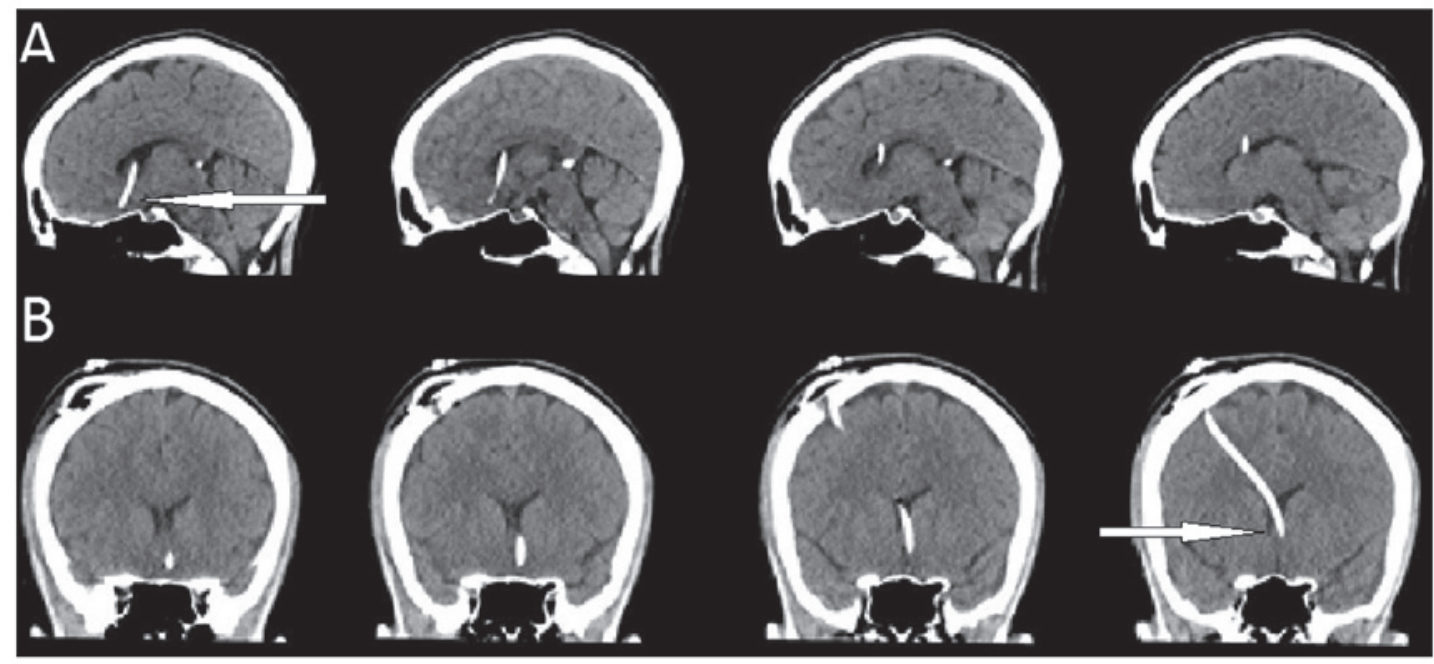

Figure 1. Head CT obtained the day after Ommaya reservoir placement showing the tip of the right-sided catheter projecting approximately 1.5 cm inferior to the floor of the left frontal ventricle. (A) Sagittal CT scan showing displaced catheter tip. (B) Coronal CT scan showing displaced catheter tip. An arrow indicates the catheter position.

left frontal ventricle (Fig. 1). Despite its deep positioning, the decision was made to use the catheter without moving it since normal CSF flow had been observed.

One day after the Ommaya placement, the patient underwent her second intrathecal administration of chemotherapy. A butterfly needle was inserted in the Ommaya dome and $15 \mathrm{ml}$ of pinkish CSF was withdrawn using a $10 \mathrm{cc}$ syringe. This was followed by administration of $15 \mathrm{mg}$ of methotrexate mixed in with $50 \mathrm{mg}$ of hydrocortisone. The procedure was carried out over $5 \mathrm{~min}$, with the drugs being flushed from the tubing with the patient's autologous CSF. At the end of the infusion the patient started developing a frontal headache, followed by nausea and an episode of emesis and diarrhea. Vital signs were stable during that episode. The fluid's pink color was attributed to blood products from recent surgery still present in the CSF spaces. CSF cytology was negative for malignancy. The post-infusion symptoms were felt to be due to a reaction to the medication and the plan going forward was to continue biweekly intrathecal chemotherapy treatments with prophylactic anti-nausea and pain medication if symptoms recurred. Five days later, the patient received her next intrathecal chemotherapy via the Ommaya reservoir. A 25-guage butterfly needle was inserted in the Ommaya dome and a small $5 \mathrm{cc}$ syringe was used for very gentle aspiration given a low platelet count as well as prior complications. Clear fluid $(8 \mathrm{ml})$ was withdrawn without complications, followed by administration of $15 \mathrm{mg}$ of methotrexate and $50 \mathrm{mg}$ of hydrocortisone, for a total volume of $5 \mathrm{ml}$. The drug was pushed over $5 \mathrm{~min}$. Close to the conclusion of the injections, the patient started developing a frontal headache and before and then at the conclusion of the injection the patient experienced several episodes of emesis. She was then medicated with lorazepam and ondansetron. It was unclear at the time whether the etiology of the adverse symptoms was mechanical or chemical in nature.

Three days later, a third dose was attempted via the Ommaya reservoir. This time, modifications were made such that the patient was pre-medicated with lorazepam and ondansetron. A $3 \mathrm{cc}$ syringe as opposed to a $5 \mathrm{cc}$ syringe was used to decrease the pressure of injection. A 23-guage butterfly needle was inserted in the Ommaya dome and $3 \mathrm{cc}$ syringes were used to withdraw the fluid. Then, $12 \mathrm{ml}$ of fluid was gradually withdrawn, followed by a very slow chemotherapy injection of $1 \mathrm{ml}$ per $2 \mathrm{~min}$. At approximately $2 \mathrm{ml}$ into the injection, the patient started experiencing a frontal headache again. Another $0.5 \mathrm{ml}$ was injected and the patient started feeling nauseous. At this point, the injection was aborted and the needle was withdrawn after flushing the tubing with $2 \mathrm{ml}$ of CSF. The patient went on to vomit and had an episode of diarrhea.

The head CT images were reviewed, and there was concern that the location of the catheter could be playing a role in the patient's adverse reaction after each intrathecal injection. It was thought that possibly changing the location of the Ommaya catheter would improve tolerance of chemotherapy. The decision was made to return to the operating room for a revision of the Ommaya catheter. The Ommaya reservoir was felt to be intact. As optimal placement would be approximately $2 \mathrm{~cm}$ shorter for the distal catheter location, the Ommaya reservoir was gently lifted out of the bur hole and held in place using a rubber shod-covered hemostat. The suture around the Ommaya reservoir was cut, and then the Ommaya reservoir itself was disconnected. The ventricular catheter was slowly advanced out of the bur hole until $22 \mathrm{~mm}$ was excised from the proximal end of the catheter. At this point, the Ommaya reservoir was reconnected and secured. Compression of the Ommaya reservoir did reveal excellent flow in and out of spinal fluid. Head CT imaging the following day verified a successful repositioning (Fig. 2).

Four weekly doses were administered following the Ommaya revision. In each case, a 23 gauge butterfly needle was inserted in the Ommaya dome and $15 \mathrm{ml}$ of clear CSF was withdrawn. This was followed by the administration of $15 \mathrm{mg}$ of intrathecal cytarabine in a total volume of $5 \mathrm{ml}$. The drug was pushed at a rate of $1 \mathrm{ml} / \mathrm{min}$. The drug was flushed from the tubing with autologous CSF to the amount of $5 \mathrm{ml}$. Contrary to previous instances, the patient tolerated all procedures well without complications. More specifically, 

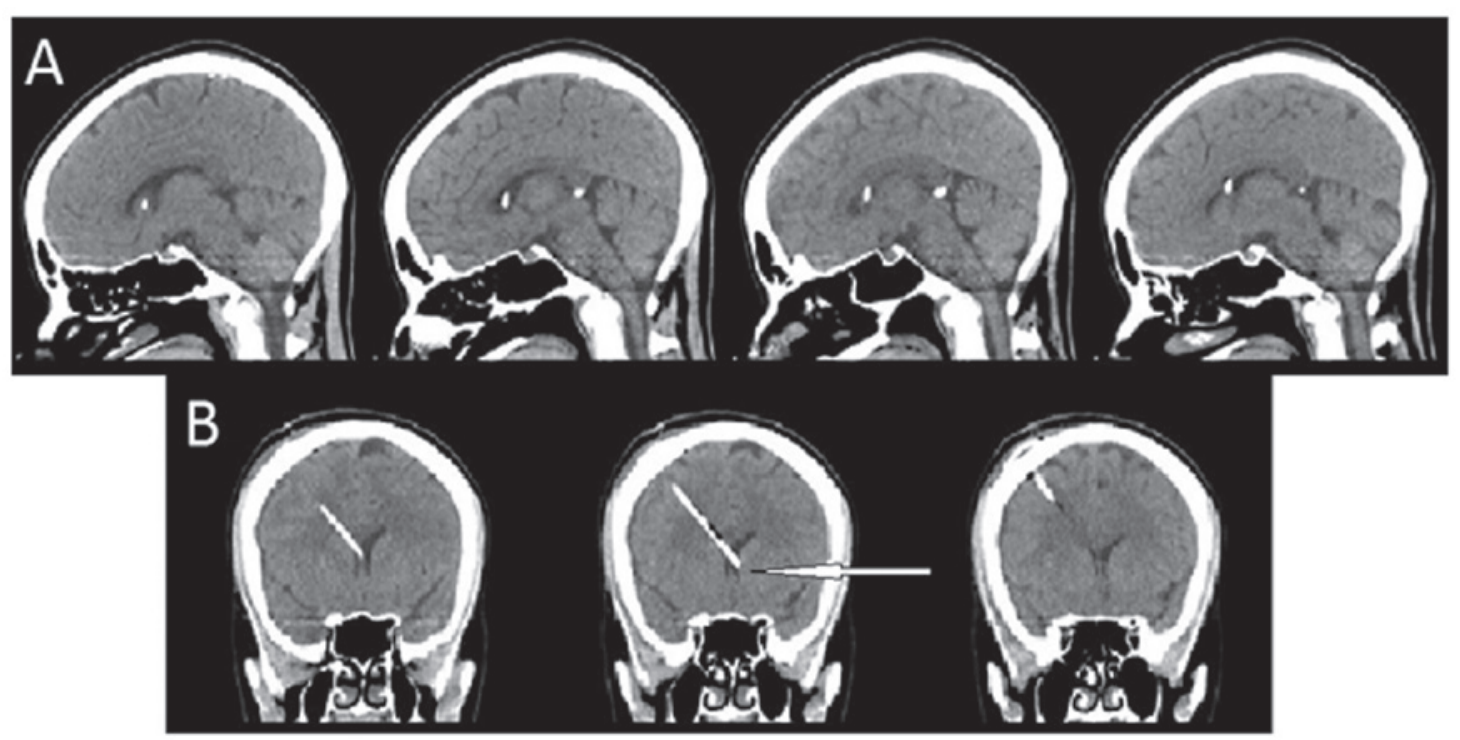

Figure 2. Head CT imaging post-revision day 1, verifying successful correction of Ommaya reservoir placement. (A) Sagittal CT images showing correctly placed catheter tip. (B) Coronal CT images showing correctly placed catheter tip. An arrow indicates the catheter position.

the reproducible frontal headache, nausea, emesis and diarrhea previously seen were completely absent.

\section{Discussion}

The patient's original adverse symptoms can almost certainly be attributed to catheter misplacement, given the fact that the symptoms improved after repositioning the catheter. However, we can only hypothesize what the pathophysiological explanation may have been. Side effects of intrathecal methotrexate toxicity are well documented in the literature, but do not match the patient's experience. Acute neurotoxicity due to intrathecal methotrexate overdose typically presents within $24-48 \mathrm{~h}$ as chemical arachnoiditis with symptoms that include headache, nuchal rigidity, back pain, vomiting, fever, and CSF pleocytosis $(9,10)$. Other documented side effects of intrathecal chemotherapy toxicity in general include spinal cord lesions (manifesting most commonly as tetraplegia, paraplegia, and cauda equina syndrome), seizure, and encephalopathy (11). Furthermore, methotrexate has a half-life in CSF on the order of hours (11). It seems unlikely that adverse symptoms due to a toxic concentration within the parenchyma would subside within minutes, as experienced by the patient. Rather, we consider the proximity of the catheter tip to the hypothalamus as a better explanation. The nucleus tractus solitarius (NTS) is central to the vagal neurocircuits involved in nausea and vomiting, and exchanges both inputs and outputs with the hypothalamus (12).

Irrespective of the underlying pathophysiology, the case demonstrates the value in troubleshooting Ommaya reservoir complications rather than prematurely abandoning its use in favor of lumbar puncture, which is also associated with adverse complications (i.e., post-LP headache, CSF leak, and discomfort to the patient). It should be noted that lumbar puncture allows the patient to avoid surgery and potential infection risk associated with the presence of a permanent catheter. However, an Ommaya reservoir was deemed appropriate in this case due to the patient's severe spinal headache following her initial lumbar puncture, as well as her requirement for repeated intrathecal chemotherapy delivery. Using an Ommaya reservoir minimizes patient discomfort and takes advantage of the natural flow of CSF, providing a more direct delivery and even distribution of chemotherapy along the CNS axis as compared to lumbar puncture (5).

The case also demonstrates the profound effects that can be caused from a small displacement of any sort of catheter within the brain. In other words, this case is of an Ommaya reservoir, these complications can arise from a small misplacement of an external ventricular device, a catheter-delivering tissue plasminogen activator, or any other catheter placed within the brain. Utmost care was taken to ensure precise initial placement of the catheter, with stereotactic trajectories planned and followed with the aid of stealth navigation registered with MRI scans taken within 3 days of surgery. Movement of catheters post-placement is a known complication, with up to $33 \%$ of misplaced ventricular catheters showing movement between intraoperative image guidance and the first post-operative scan (13).

In conclusion, both initial precision in placement and continued monitoring of catheter location can lead to drastic differences in patient care and outcomes. For this reason, catheter location should be verified with repeated imaging before and potentially between intrathecal chemotherapy infusions, especially when there are unexpected or disproportionate negative side effects from medications delivered through the device.

\section{Ackowledgements}

We would like to express our gratitude to the patient for letting us present and publish her case. Publication of this study was supported by the Comprehensive Neuro-Oncology Program Fund at Mayo Clinic, Arizona.

\section{Funding}

Not applicable. 


\section{Availability of data and materials}

Not applicable.

\section{Authors' contributions}

MMM was responsible for the article concept. CVS acquired imaging. The draft of the manuscript was prepared by DJM. All authors contributed to the design of the work and participated in the critical revision of the manuscript. All authors read, approved the final version and agreed to be accountable for all aspects of the work.

\section{Ethics approval and consent to participate}

IRB approval not required.

\section{Patient consent for publication}

Written consent obtained.

\section{Competing interests}

Not applicable.

\section{References}

1. Cohen-Pfeffer JL, Gururangan S, Lester T, Lim DA, Shaywitz AJ, Westphal $\mathrm{M}$ and Slave I: Intracerebroventricular delivery as a safe, long-term route of drug administration. Pediatr Neurol 67: 23-35, 2017.

2. Mead PA, Safdieh JE, Nizza P, Tuma S and Sepkowitz KA: Ommaya reservoir infections: A 16-year retrospective analysis J infect 68: 225-230, 2014.

3. Lishner M, Perrin RG, Feld R, Messner HA, Tuffnell PG, Elhakim T, Matlow A and Curtis JE: Complications associated with Ommaya reservoirs in patients with cancer. The Princess Margaret Hospital experience and a review of the literature. Arch Intern Med 150: 173-176, 1990.
4. Bin Nafisah S and Ahmad M: Ommaya reservoir infection rate: A 6-year retrospective cohort study of Ommaya reservoir in pediatrics. Childs Nerv Syst 31: 29-36, 2015.

5. Montes de Oca Delgado M, Cacho Diaz B, Santos Zambrano J, Guerrero Juárez V, López Martínez MS, Castro Martínez E, Avendaño Méndez-Padilla J, Mejía Pérez S, Reyes Moreno I, Gutiérrez Aceves A and González Aguilar A: The comparative treatment of intraventricular chemotherapy by ommaya reservoir vs. Lumbar puncture in patients with leptomeningeal carcinomatosis. Front Oncol 8: 509, 2018.

6. Lau JC, Kosteniuk SE, Walker T, Iansavichene A, Macdonald DR and Megyesi JF: Operative complications with and without image-guidance: A systematic review and meta-analysis of the Ommaya reservoir literature. World Neurosurg 122: 404-414, 2019.

7. Sundaresan N and Suite ND: Optimal use of the Ommaya reservoir in clinical oncology. Oncology (Williston Park) 3: 15-22; discussion 23, 1989.

8. Papanastasiou L, Fountoulakis S, Pappa T, Liberopoulos K, Malliopoulos D, Markou A and Piaditis G: Brain and optic chiasmal herniation following cabergoline treatment for a giant prolactinoma: Wait or intervene? Hormones (Athens) 13: 290-295, 2014.

9. Livshits Z, Rao RB and Smith SW: An approach to chemotherapy-associated toxicity. Emerg Med Clin North Am 32: 167-203, 2014.

10. Kerr JZ, Berg S and Blaney SM: Intrathecal chemotherapy. Crit Rev Oncol Hematol 37: 227-236, 2001.

11. Kwong YL, Yeung DY and Chan JC: Intrathecal chemotherapy for hematologic malignancies: Drugs and toxicities. Ann Hematol 88: 193-201, 2009.

12. Babic T and Browning KN: The role of vagal neurocircuits in the regulation of nausea and vomiting. Eur J Pharmacol 722: 38-47, 2014.

13. Whitehead WE, Riva-Cambrin J, Wellons JC III, Kulkarni AV, Browd S, Limbrick D, Rozzelle C, Tamber MS, Simon TD, Shannon CN, et al: Factors associated with ventricular catheter movement and inaccurate catheter location: Post hoc analysis of the hydrocephalus clinical research network ultrasound-guided shunt placement study. J Neurosurg Pediatr 14: 173-178, 2014. 